\begin{tabular}{|c|l|}
\hline Title & $\begin{array}{l}\text { Study on the Sliding Friction of Endothelial Cells Cultured on Hydrogel and the Role of Glycocalyx on Friction } \\
\text { Reduction }\end{array}$ \\
\hline Author(s) & $\begin{array}{l}\text { Chen, Y ong Mei; Kurokawa, Takay uki; Tominaga, Taiki; Y asuda, Kazunori; O sada, Y oshihito; Gong, Jian Ping; } \\
\text { Y amamoto, Kimiko; A ndo, Joji }\end{array}$ \\
\hline Citation & $\begin{array}{l}\text { Advanced Engineering Materials, 12(11), B628-B636 } \\
\text { https://doi.org/10.1002/adem.201080021 }\end{array}$ \\
\hline Issue Date & 2010-11-17 \\
\hline Doc URL & http://hdl.handle.net/2115/44824 \\
\hline Type & article (author version) \\
\hline File Information & Friction of endothelial cells 05-25.pdf \\
\hline
\end{tabular}

Instructions for use 


\title{
Study on the Sliding Friction of Endothelial Cells Cultured on Hydrogel and the Role of Glycocalyx on Friction Reduction
}

\author{
Yong Mei Chen ${ }^{\mathrm{a}, \mathrm{b}}$, Takayuki Kurokawa ${ }^{\mathrm{b}}$, Taiki Tominaga ${ }^{\mathrm{b} \dagger}$, Kazunori Yasuda ${ }^{\mathrm{c}}$, \\ Yoshihito Osada $^{\mathrm{b} \dagger}$, Jian Ping Gong* ${ }^{\mathrm{b}}$, Kimiko Yamamoto ${ }^{\mathrm{d}}$ and Joji Ando ${ }^{\mathrm{d}}$
}

${ }^{a}$ MOE Key Laboratory for Non-equilibrium Condensed Matter and Quantum Engineering, Department of Chemistry, School of Science, Xi'an Jiaotong University, Xi'an 710049, P. R. China.

${ }^{\mathrm{b}}$ Faculty of Advanced Life Science, Hokkaido University, Sapporo 060-0810, Japan

${ }^{\mathrm{c}}$ School of Medicine, Hokkaido University, Sapporo 060-0810, Japan

${ }^{\mathrm{d}}$ Graduate School of Medicine, University of Tokyo, Tokyo 113-0033, Japan

${ }^{\dagger}$ Current address: RIKEN 2-1, Hirosawa, Wako, Saitama 351-0198, Japan

*Corresponding authors: J. P. Gong, gong@ @ sci.hokudai.ac.jp, Tel \& Fax: 81-11-706-2774 


\begin{abstract}
In this study, we investigated the sliding friction of human umbilical vein endothelial cell (HUVEC) monolayer cultured on poly(sodium $p$-styrene sulfonate) (PNaSS) gel, intending to elucidate the role of the glycocalyx on the surface of endothelial cell (EC) in friction reduction. Three sets of HUVEC monolayers were investigated: 1) as-cultured HUVEC monolayer, 2) HUVEC monolayer treated by transforming growth factor $\beta_{1}$ (TGF- $\beta_{1}$ ), which increased glycocalyx by $148 \%, 3$ ) HUVEC monolayer treated by heparinase I, which reduced glycocalyx by $57 \%$, both were compared with that of the as prepared one. When being slid on flat glass surface, the frictional stress of HUVEC monolayer decreased in the order of heparinase I-treated $>$ as-cultured $>$ TGF- $\beta_{1}$-treated samples. The results suggested that glycocalyx may play a role in reducing the friction of endothelial cell monolayer.
\end{abstract}

Keywords: Friction, Endothelial cell, Glycocalyx, Gel scaffold, Lubrication 


\section{Introduction}

It is interesting to observe that red blood cells $(8 \mu \mathrm{m})$ deform and smoothly squeeze through capillaries with a substantially smaller diameter $(5 \sim 6 \mu \mathrm{m})$ than their own. In this process, friction should occur between soft endothelial cells (ECs) (Young's modulus: 0.2 8 kPa)[1,2] which cover on the inner surface of blood vessel as a monolayer, and the deformable red blood cells $(\mathrm{RBCs})(\sim 0.1 \mathrm{~Pa})[3]$. ECs are shielded from direct exposure to flowing blood by a layer of macromolecules known as the glycocalyx [4-10], which has an evenly distributed, discrete, bush-like structure of thickness $0.5 \sim 3 \mu \mathrm{m}$, greatly exceeds that of the ECs $(\sim 0.2 \mu \mathrm{m})$ themselves [8]. The glycocalyx is a negatively charged layer composed of glycoproteins and proteoglycans $[9,10]$ and performs a wide variety of critical functions in the fluid environment, such as the mechano-transduction of shear stress [5,11], exclusion of red blood cells from the EC monolayer [12-15], modulation of leukocyte attachment and rolling [16-18], and inhibition of platelet adhesion [19]. However, to our knowledge, the tribological role of the glycocalyx is unclear.

Apparently, to ensure that RBCs smoothly squeeze through the capillaries, the surface friction between the EC monolayer and the RBCs should be low. In fact, the frictional shear stress exerted on the wall of blood vessel is $\sim 2 \mathrm{~Pa}$ [20] under the static pressure of $\sim 3 \mathrm{kPa}$ for capillary [21].

Low sliding friction has also been observed between other biological soft tissues. For example, the cartilages of animal joints have a friction coefficient in the range of $0.001 \sim 0.03$ [22], which is remarkably low even for hydrodynamically lubricated journal bearings. These fascinating tribological properties of biological systems arise due to the soft and wet nature of the tissues. The friction between solids obeys Amonton's law (1699), $F=\mu W$, which says that the frictional force $F$ is linearly proportional to load $W$, and does not depend on apparent contact area $A$ of two solid surfaces and sliding velocity $v$. A hydrogel consists of a cross-linked polymer network that is solvated with water, and it therefore has a soft and wet 
nature identical to that of living tissue. From this viewpoint, the frictional behaviors of various kinds of hydrogels have been extensively studied using a tribometer and rheometer and by the surface force balance technique [23-30]. We have reported that the gel friction does not simply obey this Amonton's law, and the frictional behaviors of hydrogels are very rich and complex. For example, using a tribometer and rheometer, it has been found that hydrogel friction depends on the chemical structure of the gel, the surface properties of the sliding substrates, and the measurement conditions such as normal force, sliding velocity, sample size, and sample thickness. The friction coefficient of gels, $\mu$ changes across a wide range and exhibits very low values $\left(\mu \approx 10^{-3}-10^{-4}\right)$ that are substantially lower than those between two solid materials. Furthermore, the presence of non-adhesive dangling chains on the gel surface dramatically reduces the friction, and the brush-like gels exhibit a friction coefficient as low as $10^{-4}[23]$.

In this study, we investigate the tribological role of the glycocalyx on the surface of ECs in a fluid environment. Glycocalyx can be considered as a hydrogel layer with negative charges, because it is a negatively charged macromolecules composed of glycoproteins and proteoglycans $[11,12]$ whose polymer volume fraction has been theoretically estimated to be $1 \% \sim 2 \%[31,32]$. We assume that the glycocalyx is crucial for the surface friction of the EC monolayer. To verify this hypothesis, we first established a system to measure the sliding friction of a living human umbilical vein endothelial cell (HUVEC) monolayer cultivated on soft and wet hydrogel surface against a flat glass substrate immersed in protein-free Humedia-EB2 medium (HEM). The frictional behavior of three sets of HUVEC monolayer were investigated: 1) as-cultured HUVEC monolayer, 2) transforming growth factor $\beta_{1}$ (TGF- $\beta_{1}$ )-treated HUVEC monolayer, which increased glycocalyx by $148 \%$, 3) heparinase I-treated HUVEC monolayer, which reduced glycocalyx by $57 \%$, both were compared with that of the as prepared one.

Most in vitro studies on anchorage-dependent mammalian cells such as ECs are performed 
using cells cultured on hard and dry substrates such as cover glasses and polystyrene plates. However, when ECs cultured on a hard substrate are slid against another hard surface under normal stress, they are easily destroyed due to the stress concentration. Therefore, it is not possible to study the tribological properties of EC monolayers cultured on hard and dry scaffolds. The cultivation of EC monolayers on soft and wet hydrogels, which have a three-dimensional network structure and viscoelasticity similar to that of the macromolecular-based extracellular matrix $(\mathrm{ECM})$ in biological tissues, is an optimum strategy for overcoming this problem. In this study, we cultured a continuous EC monolayer on poly(sodium $p$-styrene sulfonate) (PNaSS) gel $[33,34]$ that contains $c . a .93 \mathrm{wt} \%$ water and has a Young's modulus of $59.4 \mathrm{kPa}$. This study is helpful for understanding the low friction mechanism observed in vascular systems and for designing artificial blood vessels with low friction surfaces.

\section{Materials and methods}

\subsection{Materials}

$N, \quad N^{\prime}$-methylenebis-(acrylamide) (MBAA; Tokyo Kasei Kogyo, Tokyo, Japan), as a cross-linking agent, was purified by recrystallization from ethanol. Sodium $p$-styrene sulfonate (NaSS; Tokyo Kasei Kogyo, Tokyo, Japan), as a monomer, and 2-oxoglutaric acid (Wako Pure Chemicals, Osaka, Japan), as an initiator, were used as purchased.

\subsection{Hydrogel preparation}

Sheet-shaped PNaSS gel was synthesized by radical polymerization of an aqueous solution of 1 M monomer (NaSS), 10 mol\% cross-linker (MBAA), and 0.1 mol\% initiator (2-oxoglutaric acid) contained in the reaction cells that were formed by two parallel glass plates separated by a silicone spacer of $1.5-\mathrm{mm}$ thickness for $6 \mathrm{~h}$ (the wavelength of UV light is $365 \mathrm{~nm}$ ). After gelation, the gels were removed from the glass cells and immersed in a large volume of ion-exchanged water for 1 week. During this period, the water was changed 2 times every day 
in order to remove residual chemicals.

The $\mathrm{pH}$ and ionic strength of the solution in the gel were adjusted to 7.4 and c.a. $0.15 \mathrm{M}$, respectively, by immersing the gel in HEPES (Sigma) buffer solution (HEPES, $5 \times 10^{-3} \mathrm{M}$; $\left.\mathrm{NaHCO}_{3}, 1.55 \times 10^{-2} \mathrm{M} ; \mathrm{NaCl}, 0.14 \mathrm{M} ; \mathrm{pH}, 7.4\right)$. The above gel was denoted as HEPES buffer-equilibrated gel.

\subsection{Cell culture}

After sterilization by autoclaving $\left(120^{\circ} \mathrm{C}, 20 \mathrm{~min}\right)$, the HEPES buffer-equilibrated gel disks of radius $7.5 \mathrm{~mm}$ and thickness ca. $3 \mathrm{~mm}$ were used for HUVEC (KURSBO Biomed. Bus.). A HUVEC suspension $\left(2.26 \times 10^{4}\right.$ cells $/ \mathrm{cm}^{2}$ in $20 \%$ FBS Medium-199) was directly seeded on the gel surfaces that not been modified by any cell-adhesive proteins or peptides before cell culture. The HUVEC-loaded samples were cultured at $37^{\circ} \mathrm{C}$ in a humidified atmosphere of $5 \%$ $\mathrm{CO}_{2}$ until the cells proliferated to a monolayer $(144 \mathrm{~h})$. The details were described in a previous paper [34].

\subsection{Hydrogel characterization}

The Young's modulus $(E)$ of the PNaSS gel was measured by compressive stress-strain measurements using a tensile-compressive tester (Tensilon RTC-1310A, Orientec Co.). From the stress-strain curve, $E$ was determined by the average slope in a strain ratio range of 0 to 0.1 . $E$ of the PNaSS gel was $59.4 \mathrm{kPa}$ and $60.2 \mathrm{kPa}$ after equilibration in protein-free and $20 \%$ FBS-containing HEM, respectively, which did not obviously change because the ionic strength (c.a. $0.15 \mathrm{M}$ ) of the two media was identical. The details were described elsewhere [35].

\subsection{Glycocalyx modulation and immunofluorescence.}

Heparan sulfate proteoglycans (HSPGs) which consist of a core protein and heparan sulfate-type glycosaminoglycan, are main proteoglycans of glycocalyx $[7,8]$ To increase the amount of HSPGs on the HUVEC surface, the HUVEC monolayers were incubated for $48 \mathrm{~h}$ in $20 \%$ FBS-containing HEM with $20 \mathrm{ng} / \mathrm{ml}$ TGF- $\beta_{1}[36,37]$. On the other hand, to disrupt HSPGs, HUVEC monolayers were incubated for $4 \mathrm{~h}$ in protein-free HEM containing 1.45 
U/ml heparinase I (Sigma) [17, 38]. Detection of HSPGs was performed as follows [39]: HUVECs were chilled briefly on ice and rinsed three times with ice cold $1 \times \mathrm{PBS}$. Then the HUVECs were incubated on ice with primary heparan sulfate antibody (US Biological, Swampscott, MA), 1:200, for $1 \mathrm{~h}$, followed by ice-cold $1 \times P B S$ rinses. Secondary antibody Alexa 488 (Molecular Probes) was used at 1:200 for $1 \mathrm{~h}$. After rinsing three times with ice cold $1 \times$ PBS, immunostained samples were examined on an Olympus FluoView FV500 Laser Scanning Confocal Microscope (Olympus, BX 50). The fluorescence intensity of the TGF- $\beta_{1^{-}}$ or heparinase I-treated HUVEC monolayers relative to that of the as-cultured HUVEC monolayers corresponds to the change in the amount of HSPGs on the HUVEC surface.

\subsection{Friction measurement}

All frictional tests were performed using a rheometer (Advanced Rheometric Expansion System, "ARES," Rheometric Scientific Inc.). A schematic representation of the friction measurement of the EC monolayers is shown in Fig. 1. Disk-shaped gel of radius $7.5 \mathrm{~mm}$ with a HUVEC monolayer on one surface was used as the test sample. The bare surface of the gel was glued to the upper surface of a coaxial disk platen by using cyanoacrylate, an instant adhesive agent (Toagosei Co., Ltd.). A square, flat glass substrate $(30 \mathrm{~mm} \times 30 \mathrm{~mm})$ or disk-shaped PNaSS gel of radius $12.5 \mathrm{~mm}$ with the same HUVEC monolayer on one surface was used as the opposing substrate.

Three sets of cell-glass experiments were performed: 1) as-cultured HUVEC monolayers vs. glass, 2) TGF- $\beta_{1}$-treated HUVEC monolayer vs. glass, 3) heparinase I-treated HUVEC monolayer vs. glass. In addition, three sets of cell-cell experiments were performed: 1) as-cultured HUVEC monolayers vs. as-cultured HUVEC monolayers 2) TGF- $\beta_{1}$-treated HUVEC monolayer vs. TGF- $\beta_{1}$-treated HUVEC monolayer, 3) heparinase I-treated HUVEC monolayer vs. heparinase I-treated HUVEC monolayer.

The opposing substrates were glued to the bottom of the container with an inner radius of 60 mm, which served as the lower platen parallel to the upper platen. The test sample and 
opposing substrate were submerged in a container containing protein-free $\mathrm{HEM}\left(37^{\circ} \mathrm{C}\right)$, and the two surfaces were compressed against each other under a normal pressure $(\mathrm{P})$ of $3.3 \mathrm{kPa}$. This pressure is the minimum value to obtain stable and reproducible friction stress of this measurement system, which is similar to the pressure exerted on the capillaries (c.a. $3 \mathrm{kPa})$ [21] but much higher than the pressure acts to compress the glycocalyx in vivo [11]. After 10 min of static loading, the opposing substrate was rotated at an angular velocity $(\omega)$ of $10^{-3}$ to $10^{-2}$ $\mathrm{rad} / \mathrm{s}$, corresponding to a maximum sliding velocity $(v=\omega R)$ of $7.5 \times 10^{-6}$ to $7.5 \times 10^{-5} \mathrm{~m} / \mathrm{s}$ at the edge of the disk-shaped gel. The torque $(T(\omega))$ was detected when the opposing sliding substrate rotated with an angular velocity, $\omega$, at a strain-constant mode. Since the sliding velocity changes with the distance from the center of the axis, the obtained torque, $T(\omega)$, is an integrated value all over the plate [26].

Our previous study has shown that the frictional stress corresponding to the sliding velocity of $\omega R$ was, $\sigma(\omega R)=[(3+\alpha) T(\omega)] /(2+\alpha) \pi R^{3}$, supposing that the torque has a power relation with $\omega$ as $T(\omega) \approx \omega^{\alpha}$. Usually, $\alpha$ lies between 0 and 1 . Therefore, we use $\sigma(\omega R)=[4 T(\omega)] / 3 \pi R^{3}$ to calculate the frictional force by setting, $\alpha=1$. This gives a good approximation [26]. The details of the friction measurement were described in a previous paper [42].

\section{Results and discussion}

\subsection{Establishment of measurement method}

We previously reported that when two like-charged flat surfaces (negatively charged hydrogel vs. flat glass or two negatively charged hydrogels) are slid against each other in aqueous solution, the osmotic repulsion of the dissociated counter-ions of the charged surfaces sustains a lubricating water layer at the interface and leads to lubrication even under pressures up to the order of sub-MPa[23-28]. The EC surface is negatively charged because the glycocalyx layer has acidic glycosaminoglycans side chains, that contain many carboxyl and sulfate groups and 
contribute to its negative charge and hydration [10]. On the other hand, the $\mathrm{SiOH}$ groups on the surface of glass dissociate with an increase in the $\mathrm{pH}$ and are negatively charged at $\mathrm{pH}=7.4$ [43]. Thus, we used flat glass (surface roughness: $R_{a}=2 \mathrm{~nm}$, contact angle to water: $22^{\circ}$ ) as the opposing substrate, expecting an osmotic repulsion of the counter-ions of the interface between the HUVEC monolayer and the flat glass substrate, which will prevent non-specific adhesion of glycocalyx to the substrate.

To accurately measure the surface friction of the HUVEC monolayer by using a rheometer (Fig. 1), we need to identify suitable testing conditions under which most HUVECs remain adhered to the gel surface in the process of friction test. During this process, when the HUVEC monolayer was compressed against the opposing substrate (flat glass or identical HUVEC-monolayers) and then the opposing substrate was subsequently rotated, a shearing stress was applied to the HUVEC monolayer. Therefore, the HUVECs were easily deformed, and they were even detached from the gel surface when the testing time is long. Fig. 2A shows the percentage of HUVEC that remained adhered to the gel surface after the friction test conducted at a sliding velocity of $7.5 \times 10^{-6} \mathrm{~m} / \mathrm{s}$ against the glass substrate. Comparing to that before friction test (Figure 2B), the density of HUVEC gradually decreased with testing time. Before $800 \mathrm{~s}$, more than $80 \%$ HUVECs remain adhered to the gel surface (Fig. 2A, C). In contrast, the percentage of HUVEC was only $60 \%$ and $40 \%$, at a testing time of $1200 \mathrm{~s}$ and $1800 \mathrm{~s}$, respectively, due to high frictional stress (i.e., frictional force per unit area) between HUVEC monolayer and flat glass. As shown in Fig.2, morphology of the most of HUVECs changed from polygonal shape (Fig. 2B) to fusiform shape (Fig. 2C), and to concentric circles (Fig.2D) after friction test, indicating that the HUVECs were deformed by the frictional shear stress.

To confirm if the frictional stress of the HUVEC monolayer could be measured by the current method as shown in Fig. 1, we first measured 3 sets of samples for a short testing time of 100s, within which almost $100 \%$ of cells remain adhered on the gel surface: a) HUVEC monolayer 
vs. glass, b) bare PNaSS hydrogel vs. glass, c) ECM layer of HUVEC vs. glass. Here, the ECM layer of HUVEC is obtained by exfoliated the HUVEC monolayer from the PNaSS hydrogel scaffold by using cellular scraper, so that only ECM beneath the HUVEC remained on the surface. All the samples were equilibrated in HEM before test. As shown in Fig. 3, during sliding, the shear stresses of HUVEC monolayer and bare PNaSS hydrogel rapidly increased initially, reaching a peak of c.a. $300 \mathrm{~Pa}$ at a few seconds, then gradually decreased with the sliding time, and became constant after several tens of seconds. On the other hand, the shear stresses of ECM sample did obviously show a peak at initial sliding, and the shear stress was much higher than the other two samples, showing an unstable behavior. The frictional stresses of the three samples increased in the order of HUVEC monolayer $<$ bare PNaSS hydrogel $<$ ECM sample. These results indicate that for a short testing time, the frictional stress of the HUVEC monolayer can be accurately measured by the current method.

Based on the above results, the frictional stress, where a constant value with testing time was reached and at the same time 100\% HUVECs remained adhered on the gel surface, was adopted as dynamic frictional stress $\left(\sigma_{d y n}\right)$. In addition, the peak value of the frictional stress was adopted as the static friction stress $\left(\sigma_{\text {stat }}\right)$ that is required to start the sliding between the HUVEC monolayer and the flat glass substrate. The ratio of $\sigma_{s t a t}\left(\sigma_{d y n}\right)$ to the normal stress applied, corresponding to the static friction coefficient $\mu_{\text {stat }}$ (dynamic friction coefficient $\left.\mu_{d y n}\right)$, was estimated in the following experiment.

\subsection{Effect of glycocalyx on EC monolayer friction}

\subsubsection{Glycocalyx modulation}

The glycocalyx consists of negatively charged glycoproteins and proteoglycans [7, 8]. Of proteoglycans, the main component is heparan sulfate proteoglycans (HSPGs) (50 90wt\%), which consist of a core protein and heparan sulfate-type glycosaminoglycan, containing 
numerous negatively charged sulfate groups and binds several plasma proteins [7]. The amount of glycocalyx on the EC surface can be enhanced using TGF- $\beta_{1}$, a cytokine that stimulate heparan sulfate proteoglycans (HSPGs), the main proteoglycan of glycocalyx synthesis [37, 38]. It was reported that TGF- $\beta_{1}$ treatment can increase the HSPG content on the EC surface by more than $50 \%$. Furthermore, the glycosaminoglycan chains of a kind of HSPG synthesized by the ECs, i.e., bioglycan, were elongated by the TGF- $\beta_{1}$ treatment [38].

On the other hand, the HSPGs on the EC surfaces can be disrupted by heparinase I, an enzyme that cleaves glycosaminoglycan side-chains from HSPGs [18, 39, 40]. It was demonstrated that heparinase treatment reduced the fluorescence intensity of fluorescence-labeled wheat germ agglutinin by $27-51 \%$ [41] and the fluorescence intensity associated with the heparan sulfate antibody by $45.9 \%$ [40]. Furthermore, the thickness and negative charge of the glycocalyx were reduced by heparinase disruption [44].

In this study, we intended to modulate the amount of the glycocalyx on HUVECs by treating the as-prepared HUVEC monolayers with TGF- $\beta_{1}$ and heparinase I. To verify the extent of change in the HSPGs after treatment, the as-cultured, TGF- $\beta_{1}$-treated, and heparinase I-treated HUVEC monolayers were subjected to immunostaining analysis. Fig. 4A shows the fluorescence intensity ratios of HUVEC monolayers treated with TGF- $\beta_{1}$ or heparinase I to that of the as-cultured HUVEC monolayer. Morphology of HUVEC monolayer treated by heparinase I and TGF- $\beta_{1}$ are shown in Fig.4C and Fig.4D, respectively. Pictures of immunostained HUVEC monolayers examined by a Laser scanning confocal microscope are shown in Fig.4E, Fig.4F, and Fig.4G for untreated, heparinase I treated, and TGF- $\beta_{1}$ treated samples, respectively. The fluorescence intensity clearly decreased to $57.0 \%$ due to heparinase I treatment and increased to $148 \%$ due to TGF- $\beta_{1}$ treatment. The results demonstrated that the amount of HSPGs in the glycocalyx substantially increased and decreased after TGF- $\beta_{1}$ and heparinase I treatment, respectively. 


\subsubsection{Frictional behavior of EC monolayer}

Fig. 4B shows the typical time profiles of the frictional shear stresses of the as-cultured, TGF- $\beta_{1}$-treated, and heparinase I-treated HUVEC monolayers slid against the flat glass substrate at a sliding velocity of $7.5 \times 10^{-6} \mathrm{~m} / \mathrm{s}$. The three samples showed similar time profiles, and the frictional stress changed remarkably with glycocalyx treatment. The static $\left(\sigma_{\text {stat }}\right)$ and dynamic $\left(\sigma_{d y n}\right)$ frictional stress of HUVEC monolayer decreased in the following order: heparinase I-treated $>$ as-cultured $>$ TGF- $\beta_{1}$-treated samples. The static $\left(\sigma_{\text {stat }}, \mu_{\text {stat }}\right)$ and dynamic $\left(\sigma_{d y n}, \mu_{d y n}\right)$ frictional stress and frictional coefficient for the samples with various amount of glycocalyx are summarized in Fig. 5.

To judge if cells underwent apoptosis or not after friction test, we cultivated the cells remained adhered on the hydrogel scaffolds after friction test by placing the samples in culture dish and incubated at $37^{\circ} \mathrm{C}$ in a humidified atmosphere of $5 \% \mathrm{CO}_{2}$. We have found that the $\mathrm{ECs}$ remained adhered and spread on the gel surface after $48 \mathrm{~h}$, indicating that ECs did not undergo apoptosis, because if ECs underwent apoptosis, they would become round and detach from the hydrogel scaffolds.

For investigating the effect of glycocalyx on EC monolayer frictional behavior, surface friction behavior between two HUVEC monolayers was further studied. The results are also shown in Fig. 5. The static frictional stress $\left(\sigma_{\text {stat }}\right)$ did not change obviously with the amount of the glycocalyx, and all the 3 samples showed a similar value. On the other hand, the dynamic frictional stress $\left(\sigma_{d y n}\right)$ decreased in the following order: heparinase I-treated $>$ as-cultured $>$ TGF- $\beta_{1}$-treated samples, which is in a similar tendency with that between HUVEC and glass.

\subsubsection{Sliding velocity effect}

HUVECs were easily detached from the gel surface at a high sliding velocity. The time that 
more than $80 \%$ HUVECs remained adhered to the gel surface was denoted as $\mathrm{T}_{80 \%}$. Fig. 6 (white bar) shows $\mathrm{T}_{80 \%}$ as a function of the sliding velocity for the HUVEC slid on glass. Compared to the $\mathrm{T}_{80 \%}$ of the as-cultured HUVEC monolayer, that of the TGF- $\beta_{1}$-treated monolayers did not change obviously, while that of the heparinase I-treated monolayers obviously decreased, especially when the sliding velocity was greater than $2.37 \times 10^{-5} \mathrm{~m} / \mathrm{s}$. The $\mathrm{T}_{80 \%}$ of all three HUVEC samples rapidly decreased to less than $100 \mathrm{~s}$ when the sliding velocity was increased to $7.5 \times 10^{-5} \mathrm{~m} / \mathrm{s}$. When the sliding velocity was increased to $7.5 \times 10^{-4}$ $\mathrm{m} / \mathrm{s}$, the HUVECs were detached from the gel surface in a few seconds before the frictional stress became constant (data no shown).

Fig. 6 (oblique bar) shows $\mathrm{T}_{80 \%}$ as a function of the sliding velocity for the HUVEC slid on HUVEC. In the case of the as-cultured and TGF- $\beta_{1}$-treated HUVEC monolayers, at a sliding velocity of $7.5 \times 10^{-6} \mathrm{~m} / \mathrm{s}$, the $\mathrm{T}_{80 \%}$ of HUVEC monolayer vs. HUVEC monolayer was larger than that of HUVEC monolayer vs. glass; in contrast, when the sliding velocity was increased to $2.37 \times 10^{-5} \mathrm{~m} / \mathrm{s}$, the $\mathrm{T}_{80 \%}$ of HUVEC monolayer vs. HUVEC monolayer was less than that of HUVEC monolayer vs. glass. In the case of the heparinase I-treated HUVEC monolayers, the $\mathrm{T}_{80 \%}$ values of HUVEC monolayer vs. HUVEC monolayer at $7.5 \times 10^{-6} \mathrm{~m} / \mathrm{s}$ and $2.37 \times 10^{-5}$ $\mathrm{m} / \mathrm{s}$ were obviously less than those of HUVEC monolayer vs. glass. Furthermore, when the sliding velocity was increased to $7.5 \times 10^{-5} \mathrm{~m} / \mathrm{s}$, the $\mathrm{T}_{80 \%}$ values of HUVEC monolayer vs.HUVEC monolayer and HUVEC monolayer vs. glass were identical, regardless of TGF- $\beta_{1}$ or heparinase I treatment. The results suggested that the glycocalyx brushes on the as-cultured and TGF- $\beta_{1}$-treated HUVEC monolayers easily interpenetrated mutually at static loading (3.3 $\mathrm{kPa}, 10 \mathrm{~min}$ ), although this condition changed with changes in the sliding velocity.

The lower $\mathrm{T}_{80 \%}$ of the heparinase I-treated sample compared to other samples is associated with two effects: one is its higher frictional stress as shown in Fig. 4B, and the other is the reduced strength of EC adhesion on the gel scaffold. Li et al. reported that heparinase-treated ECs were more easily exfoliated than the as-cultured ECs from a flat glass substrate under the same shear 
stress, because HSPG disruption with heparinase decreases the size of the prominent focal adhesion that is correlated to the adhesion strength of the ECs [39].

\subsection{Discussions}

\subsubsection{Hydrogel lubrication model}

The glycocalyx can be considered as a hydrogel layer with negative charges, whose polymer volume fraction has been estimated as $1 \% \sim 2 \%$, that is, the water volume fraction of glycocalyx could be estimated as $98 \% \sim 99 \%$. Here we try to apply the hydrogel friction model to discuss the frictional behavior of ECs. In order to favor the readers who stand at the point for biomaterials science, we shortly describe the hydrogel friction due to viscose drag here, that is, hydrogel lubrication model. When a gel sliding on a solid substrate with a liquid trapped at the gel-substrate interface, the dynamic frictional stress by the viscose drag of the liquid is [26],

$$
\sigma_{d y n}=\frac{\eta v}{h+\sqrt{K_{g e l}}}
$$

Here $\eta$ is the viscosity of liquid, $v$ the sliding velocity, $h$ the thickness of the trapped liquid, $K_{g e l}$ the permeability of the gel. $K_{g e l}$ is related to the correlation length $\xi$ of the gel as $\xi=\sqrt{K_{g e l}}$, which decreases with the increase in the network cross-linking density. For a conventional 3-dimentionally cross-linked gel, $\xi$ is in the order of 1-10 nm. Equation 1 indicates that the shear flow can penetrate into the gel with a thickness of the correlation length, or the equivalent non-slippery boundary of a gel surface is located at a depth of $\xi$ from the gel surface. On the other hand, the thickness of the trapped liquid $h$ is from two possible effects: 1) The electric double layer (EDL) formation at the like-charged interface. When two like-charged surfaces approach each other, which is also the case of our system, an osmotic repulsion exists between the interfaces to form an EDL. This EDL is strongly dependent on the ionic strength or the Debye length. At the physiological condition, the Debye length is in the order of $1 \mathrm{~nm}$; 2) Drainage liquid at interface. When two surfaces approach each other under compression in a liquid, a finite drainage time is required before the surfaces getting contact. So liquid is, transiently, trapped at the interface and serves as lubrication layer. 
The drainage layer thickness $h$ is related to the drainage time $\tau$, the surface area $A$, and the compression pressure $P$ as $h=\sqrt{3 \eta A / 4 P \tau}$ [45].

\subsubsection{Discussion of RBC friction in vivo using hydrogel lubrication model}

In the in vivo system, the shear stress exerted on the wall of the capillary was found about $2 \mathrm{~Pa}$ [20]. If we assume that this dynamic shear stress is contributed by the viscose drag of the liquid entrapped at the RBC- EC interface with an average thickness $h$ and in the glycocalyx matrix with a permeability $K_{g e l}$ on the ECs surface, the apparent lubricating thickness $h+\sqrt{K_{g e l}}$ is in the order of $1 \mu \mathrm{m}$, estimated from Eq. 1 using the viscosity of the plasma $\eta=10^{-3} \mathrm{Pas}$ and $v=10^{-3} \mathrm{~m} / \mathrm{s}$ for RBC flow [46]. This value is much larger than the Debye length $(1 \mathrm{~nm})$ of the physiological environment, indicating that the EDL surrounding the EC monolayer is so thin that plays an insignificant role in friction reduction. Furthermore, the drainage layer thickness for $\mathrm{RBC}$ is estimated as $80 \mathrm{~nm}$ using the size of $\mathrm{RBC} 8 \mu \mathrm{m}$, drainage time $1 \mathrm{~s}$ (for a single $\mathrm{RBC}$ in capillary it takes $0.5 \mathrm{~s}$ for the water to escape), and pressure $10 \mathrm{~Pa}$ [11]. This estimation suggests that the dominant origin of lubrication is not due to the formation of EDL or the drainage layer, but due to the permeability of the glycocalyx matrix to liquid, that is, $h+\sqrt{K_{g e l}} \approx \sqrt{K_{g e l}}$. The apparent lubricating thickness of $1 \mu \mathrm{m}$ is much larger than the correlation length $\xi$ of conventional 3-dimentionally cross-linked gels, and it is in the same order of brush-like glycocalyx layer thickness. The result suggests that the one-end anchored glycoclayx brushes will both sustain the load and enhance the effective lubricating layer thickness owing to their easy deformation, and therefore reduce the shear resistance dramatically. Similar dramatic friction reduction effect by drafting the polymers on gel surfaces has been observed in synthetic gel systems[47,48].

The above discussion on the friction of $\mathrm{RBC}$ is in agreement with the direct observation of the RBC-EC gap by Han et al [49]. Han et al have reported when RBCs flowing through hamster 
cremaster muscle capillary, RBCs change their morphology and squeeze through the capillary in the center, there is clearly identified gap between the membrane of flowing RBCs and ECs in the capillary. The dimension of RBC-EC gap is in the order of $1 \mu \mathrm{m}$. It has been found that the RBC-EC gap is cell free and only contains plasma (and probably the glycocalyx layer), so the gap is also called a plasma layer. This plasma layer was assumed to serve as lubricating layer, contributing for RBCs smoothly flow through on EC surface. So the results discussed based on the gel friction model seems to be in agreement with this assumption.

\subsubsection{Discussion of ECs gel friction in vitro using hydrogel lubrication model}

The frictional stress observed in this work was in the order of 10 100 Pa even for the TGF- $\beta_{1}$-treated sample, which is considerably higher than the shear stress exerted on the wall of the blood vessels of the in vivo system that has a value of $2 \mathrm{~Pa}$ [20]. The high frictional stress cannot be understood by the viscose drag mechanism. In fact, if we apply Equation 1, the

apparent lubricating layer thicknesses were estimated as $\sqrt{K_{\text {gel }}}=0.058 \mathrm{~nm}, 0.25 \mathrm{~nm}, 0.022 \mathrm{~nm}$, for as-cultured, TGF- $\beta_{1}$-treated, and heparinase I-treated HUVEC monolayer slide against flat glass, respectively (using $\eta=10^{-3} \mathrm{Pas}$, sliding velocity in this friction test $v=7.5 \times 10^{-6} \mathrm{~m} / \mathrm{s}$, and average $\sigma_{d y n}=130 \mathrm{~Pa}, 30 \mathrm{~Pa}$, and $340 \mathrm{~Pa}$ for as-cultured, TGF- $\beta_{1}$-treated, and heparinase I-treated samples, respectively). These apparent lubricating layer thicknesses have no any physical meanings, indicating that it is not proper to consider that the friction is predominantly originated from viscose drag. Direct contact between the glass substrate and the glycoxalyx or the cell surface should occur, and accordingly the dominant contribution to the frictional shear stress is from this direct contact. The large difference observed in this study and in vivo might be associated with the following three reasons:

(i) Friction was tested under a normal pressure, $3.3 \mathrm{kPa}$, which is 10 to100 times higher than the pressure exerted on the EC by the red cell membrane in vivo [11]. Under such a high 
pressure, the glycocalyx layer was highly compressed, and this will lead two effects that increase the friction: 1) An increase in the contact of solid component (glycocalyx) with the opposing surface; 2) Decrease of the permeability to liquid.

(ii) Poor growth of the glycocalyx layer on the HUVECs cultured in vitro. Recent studies have found glycocalyx deficiency when the ECs are cultured in vitro[50, 51]. Damiano E R. et al showed that the glycocalyx thickness of in vitro cultured human umbilical vein was $0.03 \mu \mathrm{m}$ under standard cell culture conditions, c.a. 17 times shorter than that of microvessels in vivo $(0.52 \mu \mathrm{m})$ [50]. Although our study indicated that the amount of glycocalyx on HUVECs cultured on the negatively charged synthetic hydrogels is obviously higher than that cultured on polystyrene culture dish[34], it is not clear if the former is as much as that in vivo. As indicated by Damiano E R [50], a deficiency in glycocalyx for ECs cultured under standard cell culture conditions likewise casts doubt on the applicability of using these EC cells to many of the vascular function and pathology studies.

(iii) The hard nature of the opposing sliding substrate (flat glass) is not favorable for sustaining a large amount of water as lubrication layer at the interface. The drainage layer thickness is theoretically estimated as $100 \mathrm{~nm}$ using the lateral dimensions of platen, $7.5 \mathrm{~mm}$, the pressure $3 \mathrm{kPa}$, and the overall measurement time $2000 \mathrm{~s}$, including the preload time. This thickness probably is too thin to maintain a continuous lubricating layer at the interface, considering the possible mis-alignment between the two parallel platens of the apparatus, and some part of the sample would be in direct contact with the hard glass surface[52].

When the HUVEC monolayers are treated with TGF- $\beta_{1}$, HSPG synthesis is stimulated and the amount of HSPGs on the surface of the HUVEC monolayer increases [37, 38]. This results in an increase in the amount of glycocalyx, either by increase in the number or in the length of the brushes. This enhancement in glycocalyx will favor the water holding ability of the glycocalyx layer against the normal compression and therefore reduce the friction. On the other hand, when the HUVEC monolayers are treated with heparinase I, HSPGs are disrupted, i.e., the 
amount of HSPGs on the HUVEC monolayers decreases [18, 40]. This leads to a decrease in the glycocalyx of the HUVEC monolayers [42], and the water holding ability decreases. In addition, after been treated by heparinase I for $4 \mathrm{~h}$ in serum free culture medium, the cell-cell contact became looser and cell became a little smaller than before HUVEC treatment. Cell shape and membrane characteristics could also influence sliding properties.

Finally, we discuss the friction behavior between HUVEC monolayers. When two HUVEC monolayers are compressed against each other and a shearing stress is subsequently applied, the overlapping of the two soft HUVEC monolayers will significantly influence the frictional behavior. Mutual interpenetration of the brush-like glycocalyx between the opposing HUVEC monolayers might occur because the normal pressure is high. This mutual interpenetration will increase the resistance against shear motion, and the depth of interpenetration should increase with the contact time. This effect may explain why the static friction $\left(\sigma_{\text {stat }}\right)$ between the HUVEC monolayers did not obviously change with the amount of glycocalyx: during compression, the glycocalyx brushes on the surface of the as-cultured and TGF- $\beta_{1}$-treated HUVEC monolayers might interpenetrate mutually to give a high resistance.

\section{Conclusions}

In this study, we established a direct experimental method for investigating the surface sliding friction of EC monolayers cultured on a soft, PNaSS gel scaffold by using a rheometer, and we studied the role of the glycocalyx on the surface friction of ECs. Using a rheometer to determine the frictional behavior of the EC monolayer has several advantages such as the possibility of measuring a small (several $\mathrm{mm}$ ) living EC monolayer cultured on a flat plate, the high sensitivity in detecting extremely small frictional torque, and the possibility of performing the measurement in the culture medium.

The frictional stress on a smooth flat glass surface was found to decrease when the HUVEC monolayers were treated with TGF- $\beta_{1}$, which stimulates the synthesis of HSPGs on the 
HUVEC surface, while it increased when the HUVEC monolayers were treated with heparinase I, which disrupts HSPGs synthesis. Comparing with the as cultured and TGF- $\beta_{1}$-treated HUVECs, heparinase I-treated HUVECs are easily detached from the cell culture scaffold, because of high friction and weak focal adhesion. So under the present experimental condition, an enhancement in the amount of glycocalyx will increase the osmotic pressure of the glycocalyx layer which sustains the high pressure applied and retain more liquid in the glycocalyx gel layer, and favors the low friction. The study suggests that the glycocalyx play an important role in reducing the surface friction of ECs.

\section{Acknowledgements}

The present work was supported by Grants-in-Aid for Specially Promoted Research (No. 18002002) from the Ministry of Education, Culture, Sports, Science and Technology (MEXT), Japan. 


\section{References}

[1] Pesen D, Hoh JH. Micromechanical architecture of the endothelial cell cortex. Biophys J 2005; 88: 670-679.

[2] Shen Y, Sun JL, Zhang A, Hu J, Xu LX. A new image correction method for live cell atomic force microscopy. Phys Med Biol 2007; 52: 2185-2196.

[3]Henon S, Lenormand G, Richert A, Gallet F. Direct measurement of the area expansion and shear moduli of the human red blood cell membrane skeleton. Biophys J 1999; 75:1145-1151.

[4] Weinbaum S, Tarbell JM, Damiano ER. The structure and function of the endothelial glycocalyx layer. Annu. Rev. Biomed. Eng. 2007, 9:121-167

[5] Tarbell JM, Pahakis MY. Mechanotransduction and the glycocalyx. J Intern Med 2006; 259:339-50.

[6] Pries AR, Secomb TW, Gaehtgens P. The endothelial surface layer. Eur J Physiol 2000;440: $653-66$.

[7] Tarbell JM, Weinbaum S. Kamm RD. Cellular fluid mechanics and mechanotransduction. Annals Biomed Eng 2005;33: 1719-23.

[8] Bernard M, Berg VD, Vink H, Spaan JAE. The endothelial glycocalyx protects against myocardial edema. Circ Res 2003;92:592-4.

[9] Nieuwdorp M, Meuwese MC, Vink H, Hoekstra JBL, Kastelein JJP, Stores ESG. The endothelial cell glycocalyx: a potential barrier between health and vascular disease. Cuur Opin Lipidol 2005; 16:507-11.

[10] Sabri S, Soler M, Foa C, Anne-Marie Benoliel PA, Bongrand P. Glycocalyx modulation is a physiological means of regulating cell adhesion. J Cell Sci 2000; 113: 1589-600. 
[11] Weinbuma S, Zhang X, Han Y, Vink H, and Cowin SC. Mechanotransduction and flow across the endothelial glycoalyx. PNAS 2003; 100:7988-95.

[12] SecombTW, Hsu R, Pries AR. Motion of red blood cells in a capillary with an endothelial surface layer: effect of flow velocity. Am J Physiol Heart Circ Physiol 2001; 281:H629-36.

[13] Damiano1 ER. The effect of the endothelial-cell glycocalyx on the motion of red blood cells through capillaries. Microvasc Res 1998; 55:77-91.

[14] Feng J, Weinbaum S. Lubrication theory in highly compressible porous media: the mechanics of skiing, from red cells to humans. J Fluid Mech 2000;422: 281-317.

[15] Vink H, Duling BR. Identification of distinct luminal domains for macromolecules, erythrocytes, and leukocytes within mammalian capillaries. Circ Res 1996; 79, 581-89.

[16] Constantinescu AA, Vink H, Spaan JAE. Endothelial Cell Glycocalyx Modulates Immobilization of Leukocytes at the Endothelial Surface. Arterioscler Thromb Vasc Biol $2003 ; 23: 1541-7$.

[17] Mulivor AW, Lipowsky HH. Role of glycocalyx in leukocyte-endothelial cell adhesion. Am J Physiol Heart Circ Physiol 2002; 283:H1282-91.

[18] Zhang X, Curry FR, and Weinbaum S. Mechanism of osmotic flow in a periodic fiber array. Am J Physiol Heart Circ Physiol 2006; 290: H844-52.

[19] Vink H, Constantinescu AA, Spaan JAE. Oxidize lipoproteins degrade the endothelial surface layer implications for platelet-endothelial cell adhesion. Circulation 2000; $101: 1500-2$.

[20] Kamiya A, Bukhari R, Togawa T. Adaptive regulation of wall shear stress optimizing vascular tree function. Bull Math Biol. 1984; 46:127-137

[21] Hahn M. Shore AC. The effect of rapid local cooling on human finger nailfold capillary 
blood pressure and blood cell velocity. J Physiol 1994; 478: 109-114.

[22] McCutchen CW. The frictional properties of animal joints. Wear 1962; 5: 1-17.

[23] Gong JP. Friction and lubrication of hydrogels-its richness and complexity. Soft Matter 2006; 2: 544-552.

[24] Gong JP, and Osada Y. Gel friction: A model based on surface repulsion and adsorption. J Chem Phys 1998;109:8062-8.

[25] Gong JP, Iwasaki Y, Osada Y. Friction of gels. 3. Friction on solid surfaces. J Phys Chem B 1999;103:6001-7.

[26] Gong JP, Kagata G, Osada Y. Friction of gels. 4. Friction on charged gels. J Phys Chem B 1999;103:6007-14.

[27] Gong JP, Kagata G, Iwasaki Y, Osada Y. Surface friction of polymer gels 1. Effect of interfacial interaction. Wear 2001; 251:1183-7.

[28] Kagata G, Gong JP, Osada Y. Friction of Gels. 7. Observation of static friction between like-charged gels. J Phys Chem B 2003; 107: 10221-5.

[29] Kampf N, Raviv U, Klein J. Normal and shear forces between adsorbed and gelled layers of chitosan, a naturally occurring cationic polyelectrolyte. Macromolecules 2004; $37: 1134-42$.

[30] Chestakova A, Lau W, Kumacheva E. Shear in surface gel of associative polymer. Macromolecules 2004; 37: 5047-53.

[31] Curry FE. Determinants of capillary permeability: a review of mechanisms based on single capillary studies in the frog. Circ Res. 1986; 59:367-80.

[32] Weinbaum S, Tsay R, Curry RE. A three-dimensional junction-pore-matrix model for 
capillary permeability. Microvasc Res. 1992; 44:85-111.

[33] Chen YM, Shiraishi N, Satokawa H, Kakugo A, Narita T, Gong JP, Osada Y, Yamamoto $\mathrm{K}$, Ando J. Cultivation of endothelial cells on adhesive protein-free synthetic polymer gels. Biomaterials 2005; 26:4588-96.

[34] Chen YM, Tanaka M, Gong JP, Yasuda Y, Yamamoto S, Shimomura M, Osada Y. Platelet adhesion to human umbilical vein endothelial cells cultured on anionic hydrogel scaffolds. Biomaterials 2007; 28:1752-60.

[35]Chen YM, Gong JP, Tanaka M, Yasuda K, Yamamoto S, Shimomura M, Osada Y, Tuning of cell proliferation on tough gels by critical charge effect. J Biomed Mater Res 2009; 88A: 74-83.

[36] Kaji T, Yamada A, Miyajima S, Yamamoto C, Fujiwara Y, Wight TN, Kinsella MG. Cell density-dependent regulation of proteoglycan synthesis by transforming growth factor- $\beta_{1}$ in cultured bovine aortic endothelial cells. J Biol Chem 2000; 275:1463-70.

[37] Kasinath BS, Glomerular EC. Proteoglycan regulation by TGF- $\beta_{1}$. Archives Biochem Biophys 1993; 305:370-7.

[38] Linhardt RJ, Turnbull JE, Wang HM, Loganathan D, Gallagher JT. Examination of the substrate specificity of heparin and heparan sulfate lyases. Biochem 1990;29:2611-7.

[39] Moon JJ, Matsumoto M, Patei S, Lee L, Guan JL, Li S. Role of cell surface heparan sulfate proteoglycans in endothelial cell migration and mechanotransduction. J. Cellular Physiolo 2005;203:166-76.

[40] Florian JA, Kosky JR, Ainslie K, Pang Z, Dull RO, Tarbell JM. Heparan sulfate proteoglycan is a mechanosensor on endothelial cells. Circ Res 2003; 93:e136-42.

[41] Thi MM, Tarbell JM, Weinbaum S, Spray D. The role of the glycocalyx in recognization 
of actin cytoskeleton under fluid stress: A "bumper-car" model. PNAS 2004; $101: 16483-16488$.

[42] Kagata G, Gong JP, and Osada Y. Friction of gels. 6. Effects of sliding velocity and viscoelastic responses of the network. J Phys Chem B 2002; 106:4596-601.

[43] Shah G, Dubin PL, Kaplan JI, Newkome GR, Moorefield CN, Baker GR. Size-exclusion chromatography of carboxyl-terminated dendrimers as a model for permeation of charged particles into like-charged cavities. J. Colloid Inter. Sci. 1996; 183: 397-407.

[44] Pries AR, Secomb TW, Jacobs H. Microvascular blood flow resistance: role of endothelial surface layer. Am J Physiol 1997; 273:H2272-9.

[45] Presson BNJ, Sliding Friction: Physical Principles and Applications, 2nd ed., NanoScience and Technology Series, Springer, Berlin, 2000.

[46] Unekawa M, Tomita M, Tomita Y, Toriumi H, Miyaki K, Suzuki N, RBC velocities in single capillaries of mouse and rat brains are the same, despite 10-fold difference in body size, Brain Res 2010;1320: 69-73.

[47] Gong_ J P, Kurokawa T, Narita T., Kagata G., Nishimura G., Kinjo M, Osada Y, Synthesis of Hydrogels with Extremely Low Surface Friction, J Am Chem Soc 2001; 123:5582-3

[48] Ohsedo Y, Takashina R, Gong JP, Osada Y, Surface Friction of Hydrogels with Well-Defined Polyelectrolyte Brushes, Langmiur, 2004; 20: 6549-55.

[49] Han Y, Weinbaum S, Spaan JAE, Vink H. Large-deformation analysis of the elastic recoil of fibre layers in a Brinkman medium with application to the endothelial glycocalyx. $\mathrm{J}$ Fluid Mech. 2006; 554:217-235.

[50] Potter DR, Damiano ER. The hydrodynamically relevant endothelial cell glycocalyx observed in Vivo is absent in vitro. Circ Res 2008; 102:770-6.

[51] Jacob M, Rehm M, Loetsch M, Paul JO, Bruegger D, Welsch U, Conzen P, Becker BF. The endothelial glycocalyx prefers albumin for evoking shear stress-induced, nitric 
oxide-mediated coronary dilatation. J Vasc Res 2007; 44:435- 43.

[52] Oogaki S, Kagata G, Kurokawa T, Osada Y, Gong JP, Friction between like-charged hydrogels: combination of boundary, hydrated, and hydrodynamic lubrication mechanism, $2009 ; \quad 5: 1804-11$. 


\section{Figure captions}

Figure 1 Schematic representation of the experimental setup for friction measurement of EC monolayers cultured on PNaSS hydrogel. (1) Disk-shaped gel (radius $\mathrm{R}=7.5 \mathrm{~mm}$, thickness $\mathrm{H}$ $=3 \mathrm{~mm}$ ), (2) HUVEC monolayer cultured on the gel, (3) protein-free HEM, (4) opposing substrate (glass plate or HUVEC monolayer cultured on PNaSS gel; $\mathrm{H}=3 \mathrm{~mm}$ ). Normal stress: $3.3 \mathrm{kPa}$; temperature: $37^{\circ} \mathrm{C}$.

Figure 2 Information regarding HUVECs on the gel surface after the friction test. (A) Ratio of the number of HUVECs after and before the HUVEC monolayers were slid against the glass substrate for different times at a sliding velocity of $7.5 \times 10^{-6} \mathrm{~m} / \mathrm{s}$. The average number was determined by counting the number of HUVECs after sliding for different times. The number of HUVECs was counted using micrographs ((B) and (C)) that were taken over a region 5 7.5 $\mathrm{mm}$ from the center of the disk-shaped samples; 4 micrographs were taken per sample. The error ranges are standard derivation over $3 \sim 5$ samples. Normal stress: $3.3 \mathrm{kPa}$. (D) A representative phase-contrast micrograph of a TGF- $\beta_{1}$-treated HUVEC monolayer after it was slid against an identical HUVEC monolayer for $1200 \mathrm{~s}$ at a sliding velocity of $7.5 \times 10^{-6} \mathrm{~m} / \mathrm{s}$. Concentric circles were clearly observed. The image is that of a disk-shaped sample attached to the upper plate of the rheometer.

Figure 3 Time profiles of the frictional stress for 3 sets of samples: a) HUVEC monolayer vs. glass, b) bare PNaSS hydrogel vs. glass, c) ECM of HUVEC vs. glass. Sliding velocity: $7.5 \times$ $10^{-6} \mathrm{~m} / \mathrm{s}$.

Figure 4 Analysis of cell surface HSPGs in the HUVEC glycocalyx (A) and time profiles of frictional stress (B) of the corresponding HUVEC monolayers. (A) Fluorescence intensity ratio 
of HUVECs monolayers treated with heparinase I or TGF- $\beta_{1}$ to the as-cultured HUVEC monolayer; 5 micrographs were taken per sample. The error ranges are standard derivation over 3 4 samples. (B) Time profiles of frictional stress of as-cultured, heparinase I-treated, and TGF- $\beta_{1}$-treated HUVEC monolayers. Sliding velocity: $7.5 \times 10^{-6} \mathrm{~m} / \mathrm{s}$. Normal stress: $3.3 \mathrm{kPa}$. Morphology of HUVEC monolayer treated by heparinase I and TGF- $\beta_{1}$ are shown in C and D, respectively. Pictures of immunostained HUVEC monolayers examined on a laser scanning confocal Microscope are shown in E, F, and G for untreated, heparinase I treated, and TGF- $\beta_{1}$ treated samples, respectively. Scale bar in E, F, G:200 $\mu$ m.

Figure 5 Static $\left(\sigma_{\text {stat }}\right)$ and dynamic $\left(\sigma_{d y n}\right)$ frictional stress of HUVEC monolayer vs. glass, HUVEC vs HUVEC monolayers, for as-cultured, heparinase I-treated, and TGF- $\beta_{1}$-treated samples. The right vertical axis is the corresponding results of static $\left(\mu_{s t a t}\right)$ and dynamic $\left(\mu_{d y n}\right)$ frictional coefficient that is defined as the ratio of the frictional force to the normal load applied. Sliding velocity: $7.5 \times 10^{-6} \mathrm{~m} / \mathrm{s}$. Normal stress: $3.3 \mathrm{kPa}$.

Figure 6 Time for which more than 80\% HUVECs remained adhered to the PNaSS gel surfaces after the friction test for HUVEC monolayers sliding against a glass substrate $\left(\mathrm{T}_{80 \%}\right.$ of HUVECs-glass) and those sliding against identical HUVEC monolayers ( $\mathrm{T}_{80 \%}$ of HUVECs-HUVECs) for as-cultured, heparinase I-treated, and TGF- $\beta_{1}$-treated HUVEC monolayers. White bar: HUVECs-glass, bar with oblique lines: HUVECs-HUVECs. The average time was determined by counting the number of HUVECs after sliding different for times. The number of HUVECs was counted using micrographs that were taken over a region 5 7.5 mm from the center of the disk-shaped samples; 4 micrographs were taken per sample. The error ranges are standard derivation over 3 5 samples. Normal stress: $3.3 \mathrm{kPa}$. 


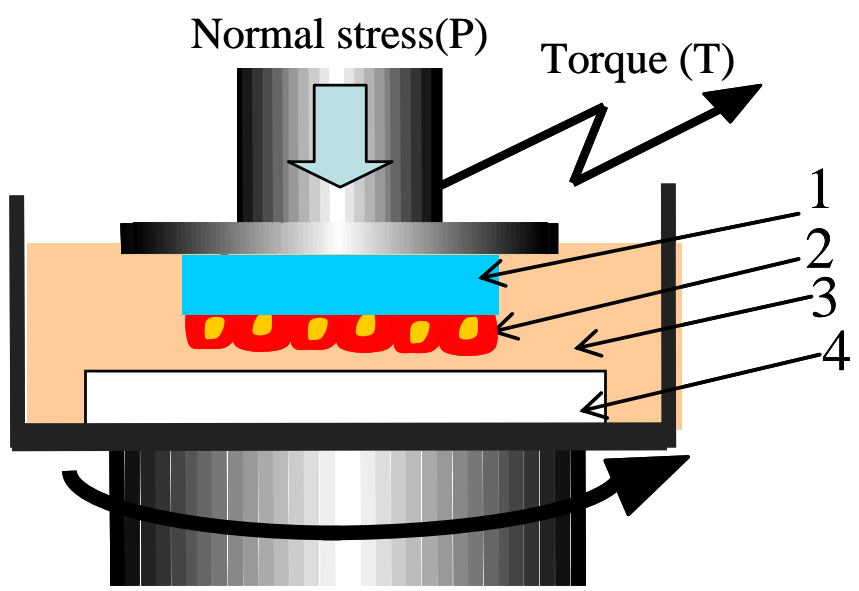

Angular velocity $(\omega)$

Fig. 1 

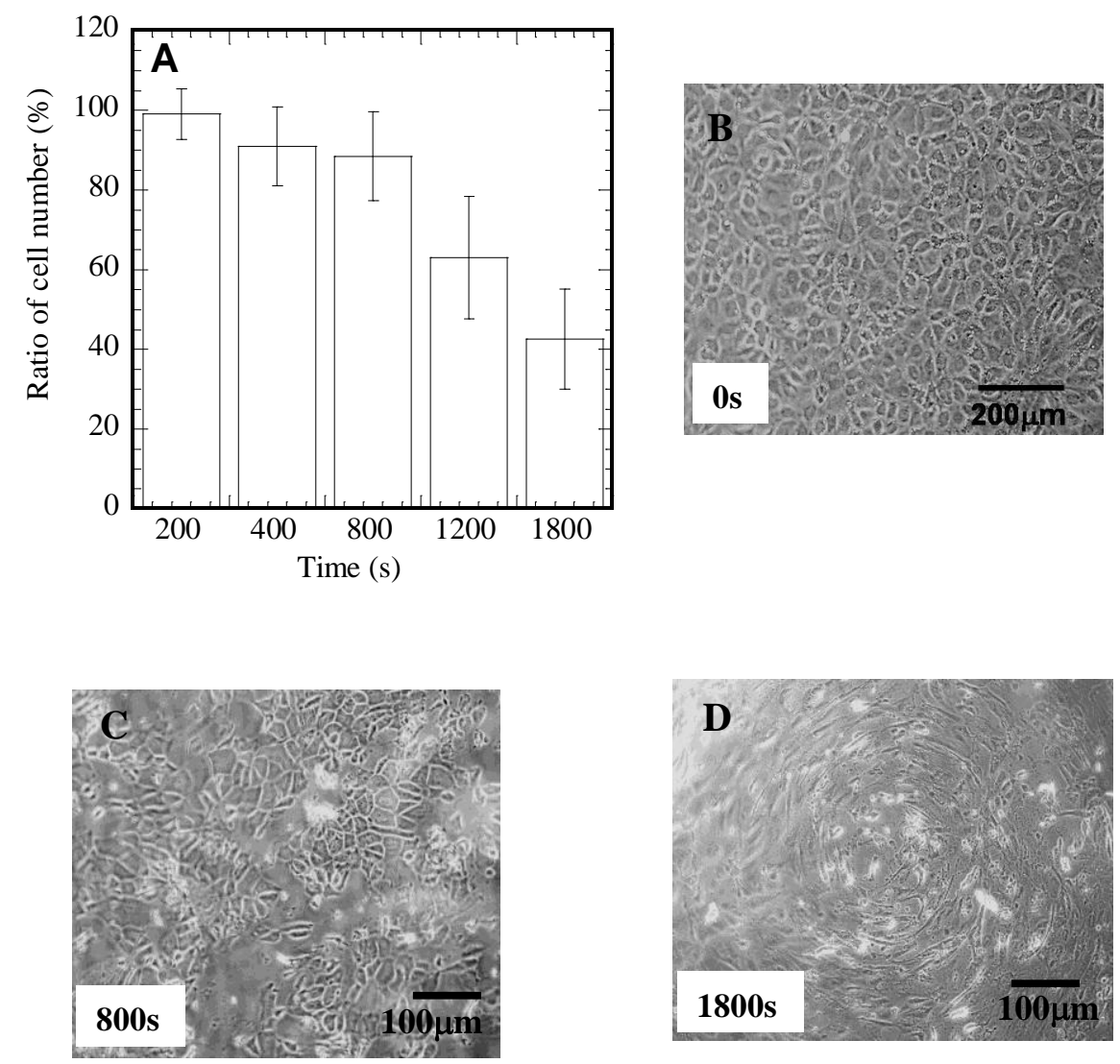

Fig. 2 


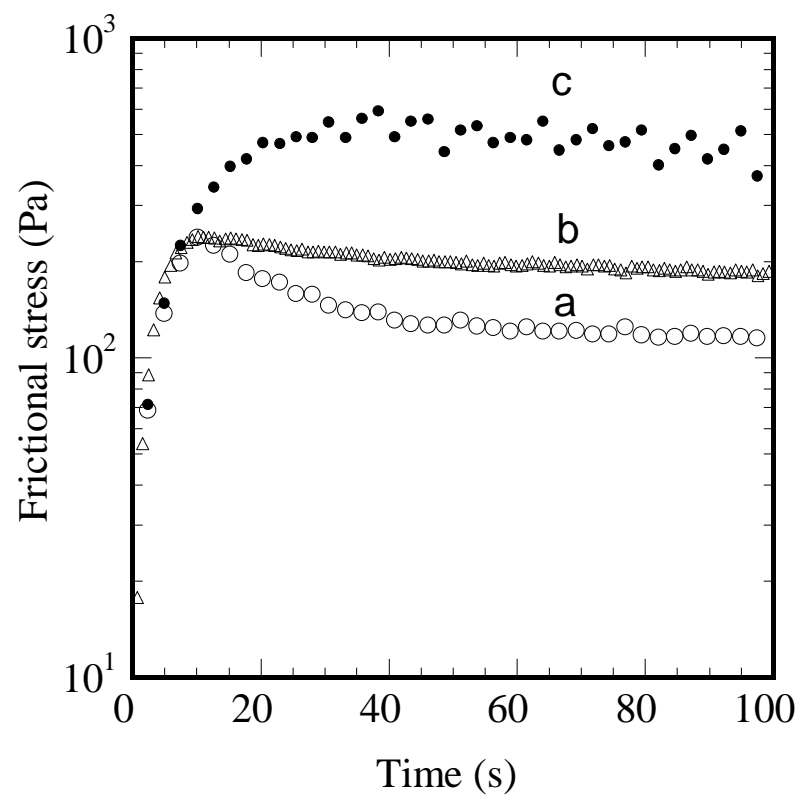

Fig. 3 

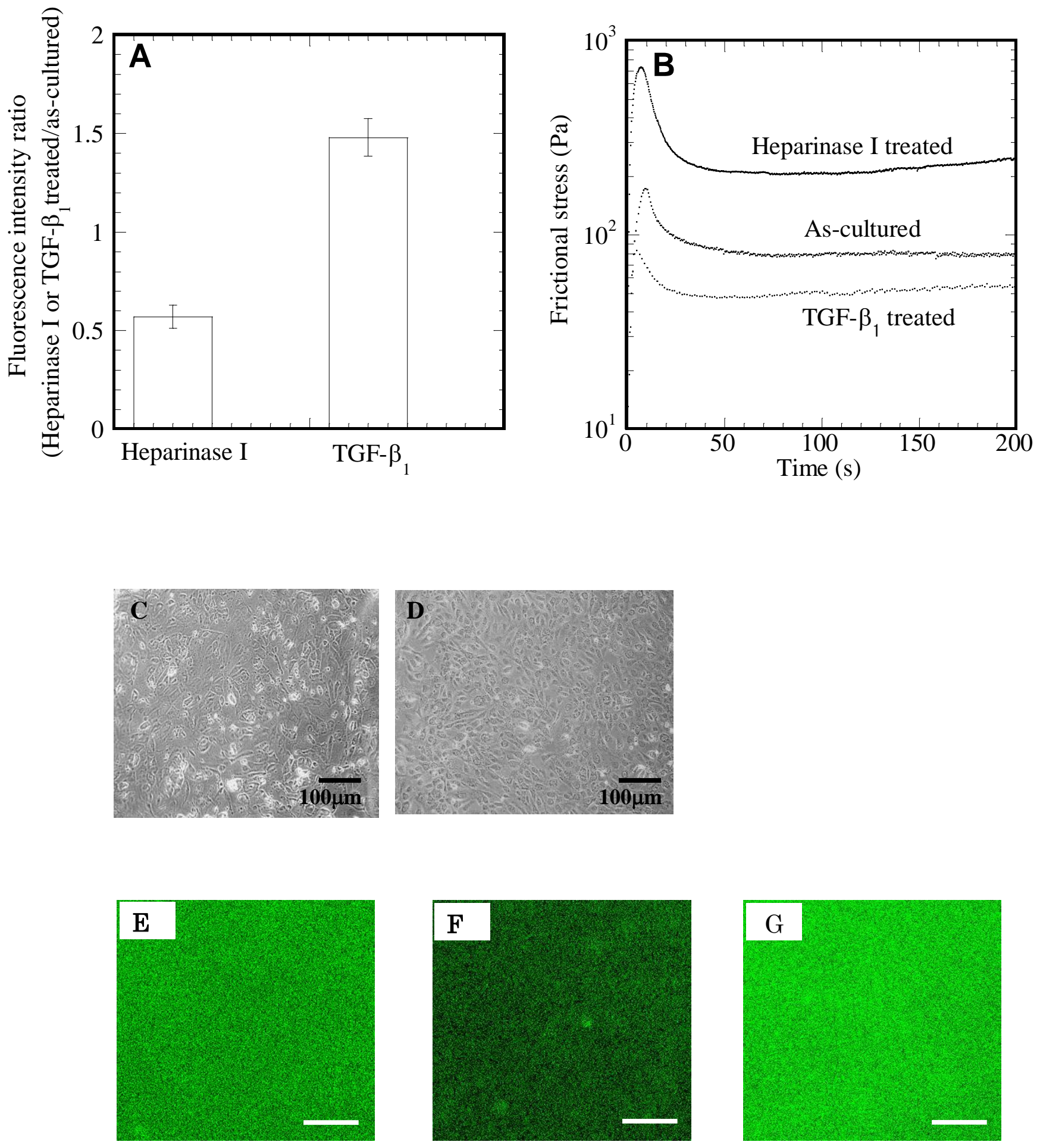

Fig. 4 


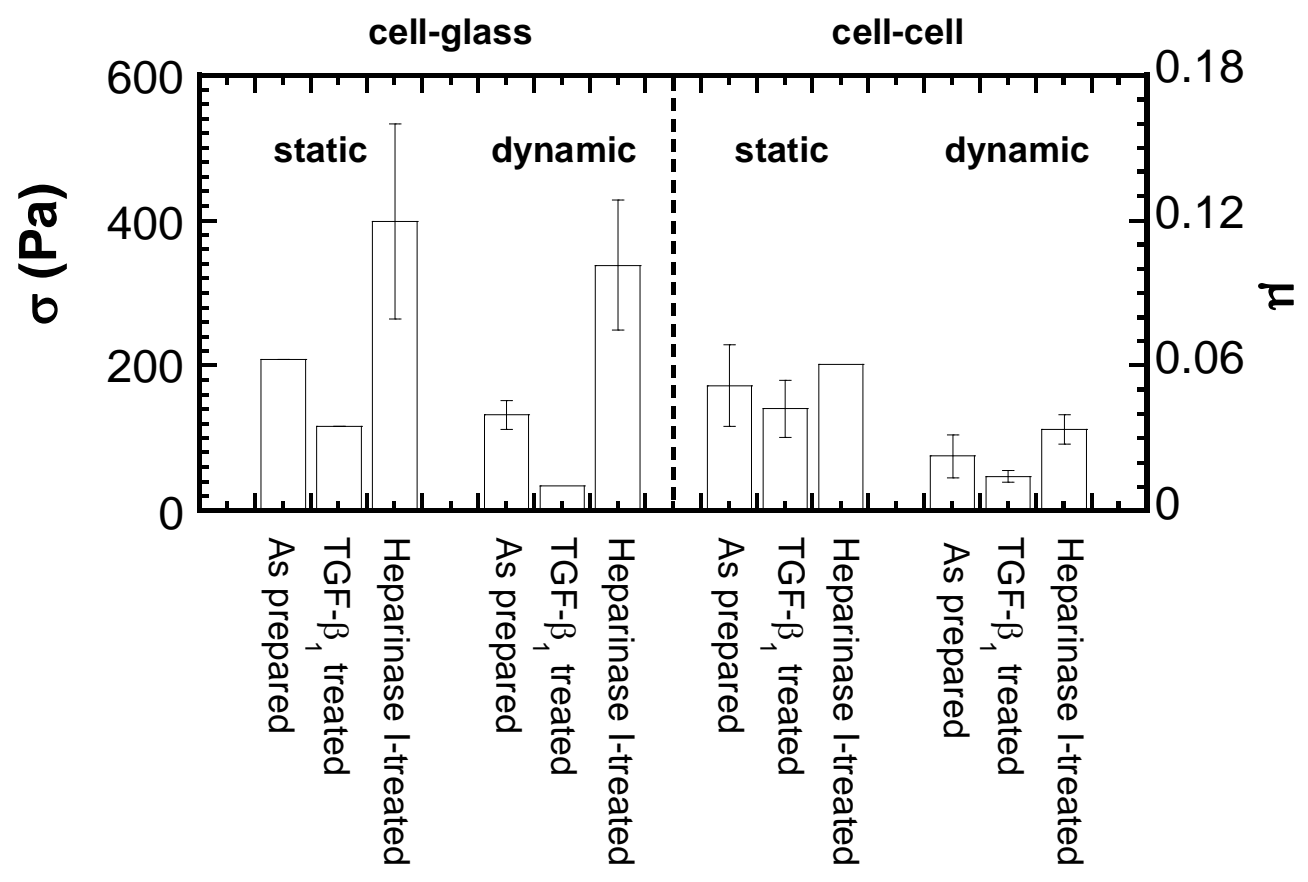

Fig. 5 


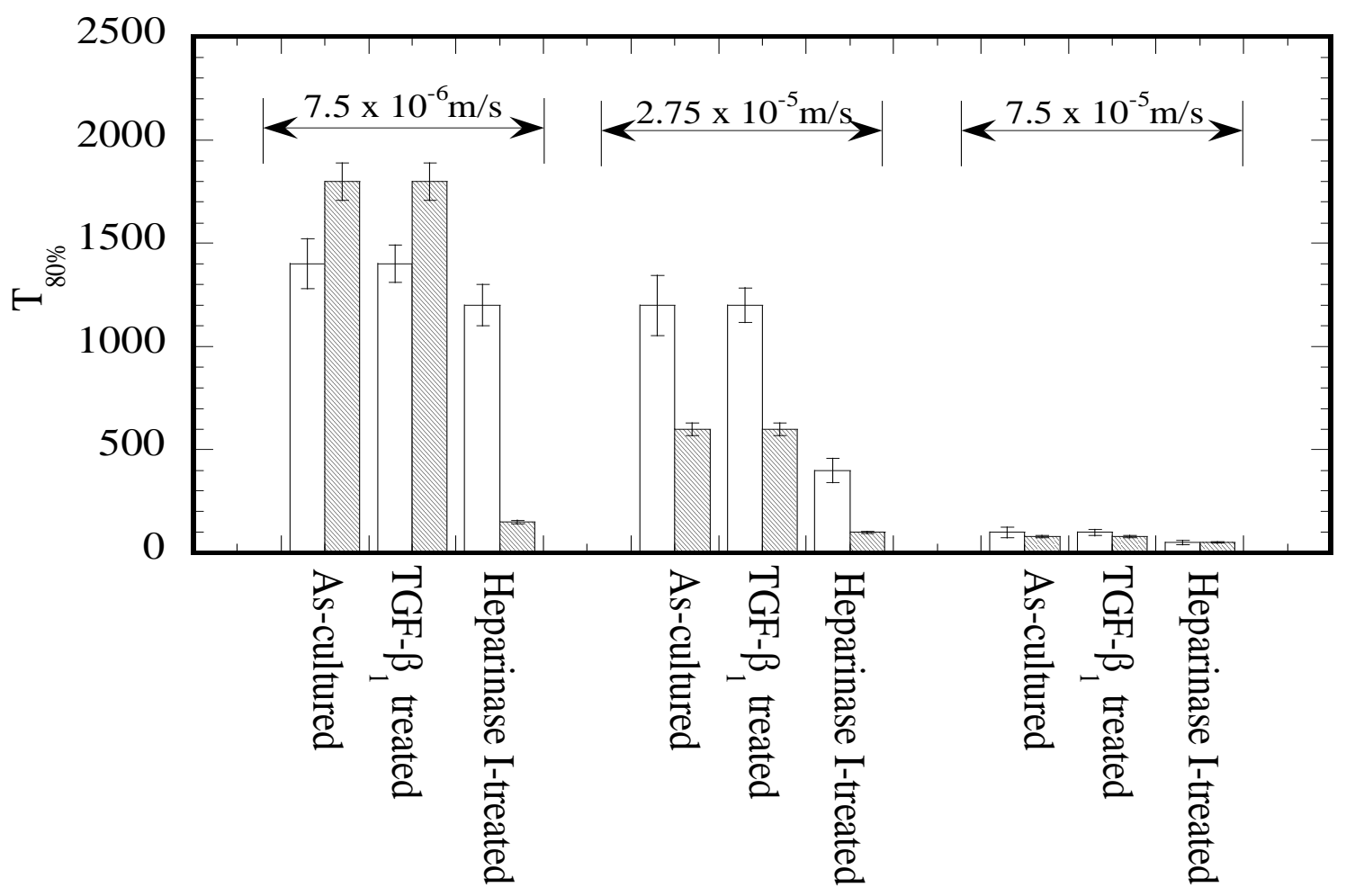

Fig. 6 
Table of Contents

\section{Study on the Sliding Friction of Endothelial Cells Cultured on Hydrogel and the Role of Glycocalyx on Friction Reduction}

Sliding friction of human umbilical vein endothelial cell (HUVEC) monolayer cultured on poly(sodium $p$-styrene sulfonate) (PNaSS) hydrogel was investigated, intending to elucidate the role of the glycocalyx on the surface of endothelial cell in friction reduction. Three sets of HUVEC monolayers were investigated: 1) as-cultured HUVEC monolayer, 2) HUVEC monolayer treated by transforming growth factor $\beta_{1}\left(\right.$ TGF- $\left.\beta_{1}\right)$, which increased glycocalyx, 3$)$ HUVEC monolayer treated by heparinase I, which reduced glycocalyx. The frictional stress of these samples against glass substrate decreased in the order of heparinase I-treated > as-cultured $>$ TGF- $\beta_{1}$-treated samples, suggested that glycocalyx may play a role in reducing the friction of endothelial cells.

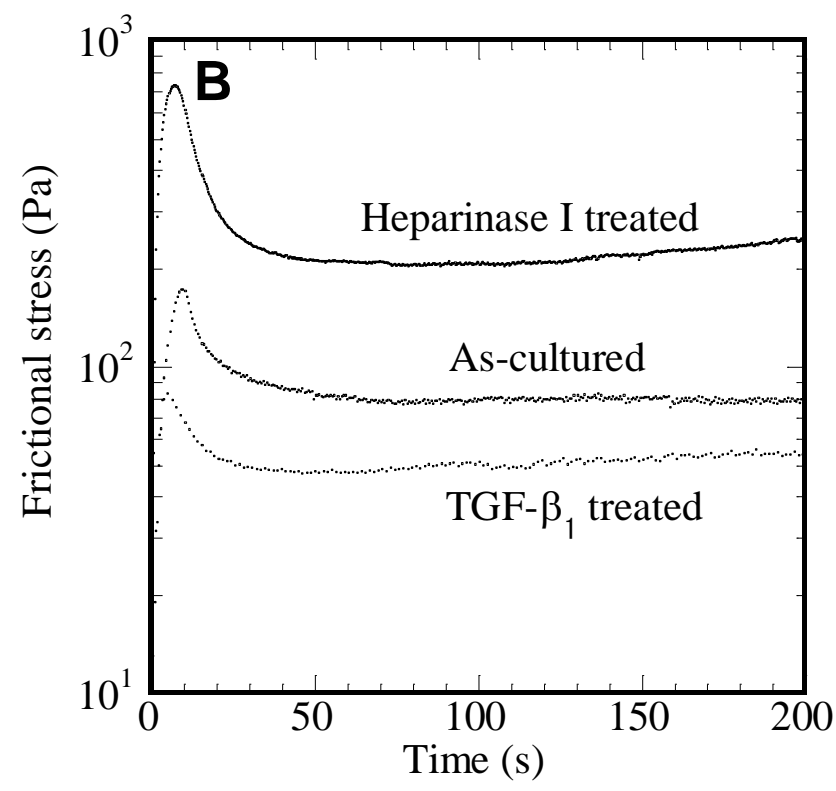

